\title{
A Dichotomy Principle for Universal Series
}

\author{
by \\ V. FARMAKI and V. NESTORIDIS \\ Presented by Jean-Pierre KAHANE
}

\begin{abstract}
Summary. Applying results of the infinitary Ramsey theory, namely the dichotomy principle of Galvin-Prikry, we show that for every sequence $\left(\alpha_{j}\right)_{j=1}^{\infty}$ of scalars, there exists a subsequence $\left(\alpha_{k_{j}}\right)_{j=1}^{\infty}$ such that either every subsequence of $\left(\alpha_{k_{j}}\right)_{j=1}^{\infty}$ defines a universal series, or no subsequence of $\left(\alpha_{k_{j}}\right)_{j=1}^{\infty}$ defines a universal series. In particular examples we decide which of the two cases holds.
\end{abstract}

Introduction. The theory of universal series was initiated by Fekete (1914) (cf. [P]), followed by Menchoff (1945) [M] (on universal trigonometric series) and Seleznev (1951) [Se]. Later, in the results by Luh (1970) [L] and Chui-Parnes (1971) [CP], the approximation by the partial sums of a universal power series holds outside the closure of the domain of definition. Nestoridis (1996) $[\mathrm{N}]$ strengthened these results, obtaining approximation on the boundary as well. There are further results on universal Faber, Jacobi, Dirichlet and Laurent series, and on harmonic expansions. We refer the reader to the two survey papers by Grosse-Erdmann (1999) [G-E] and Kahane (2000) [K].

According to an abstract theory of universality presented in $[\mathrm{NP}]$ and [BGNP], the existence of universal series is equivalent to a condition of simultaneous double approximation by a finite linear combination in a family of simple functions forming a vector space. The abstract theory covers most of the previously known cases and leads to simplification of known proofs, since the condition of double approximation follows from various classical approximation theorems (of Mergelyan, Runge, Weierstrass, Walsh etc.). At the same time the abstract approach produces several new cases of universality, such as those defined by means of the normal distribution, or in PDE's.

2000 Mathematics Subject Classification: Primary 05D10, 41A58; Secondary 30B30. Key words and phrases: universal series, Galvin-Prikry theorem. 
The theory of Ramsey infinitary combinatorics contains the dichotomy results by Nash-Williams (1965) [N-W] for open partitions, Galvin-Prikry (1973) [GP] for Borel partitions, Silver (1970) [Si] for partitions determined by analytic sets, and the result of Ellentuck (1974) [E]. Extensions involving Schreier sets have been given by Farmaki (2004) [F], and by FarmakiNegrepontis (2006) [FN1], (2008) [FN2]. These results, or others of the same type, have found important applications in various branches of mathematics, notably in Banach space theory; we refer the reader to the survey paper by Gowers (2003) [G].

In the present note we apply the Ramsey infinitary combinatorics to the theory of universal series. The meeting point is the fact, shown by an appeal to Baire's category theorem, that under very general conditions, the set of universal series is either empty, or a (dense) $G_{\delta}$ set in a suitable space $([\mathrm{G}-\mathrm{E}],[\mathrm{NP}],[\mathrm{BGNP}])$. It is precisely this fact that makes it possible to employ the Galvin-Prikry dichotomy theorem (stated in Theorem 1.7 below) to prove that every scalar sequence possesses a subsequence, all of whose subsequences are in the universal class $\mathcal{U}$, defined in Section 1 below, or all are in the complement of $\mathcal{U}$ in the space of all scalar sequences.

In Section 1 we give the definitions of the class $\mathcal{U}$, and of some classes of universal sequences more complicated than $\mathcal{U}$. In Section 2 we prove our main dichotomy result (Theorem 2.1) for these special classes of universal sequences. The fact that these classes are exactly (dense) $G_{\delta}$ subsets of specific Polish spaces, and not only residual, allows for the use of the dichotomy principle of Galvin-Prikry for suitable partitions. Crucial to the proof is the highly non-trivial result, attributed to Lusin and Suslin, mentioned in Theorem 1.6 below, according to which any 1-1 continuous image of a Borel set is Borel. In Section 3 we examine some particular concrete examples of universal series, for which we verify the general dichotomy principle in a direct, elementary way, without recourse to Ramsey theory (and to Theorem 2.1), deciding in addition which alternative actually holds.

There are a number of interesting questions on which we have no answer. It would be desirable to have an effective criterion to decide which alternative of the dichotomy holds in every specific instance, in particular, when hereditary universality actually holds.

1. Preliminaries and notation. We denote by $\mathbb{N}=\{0,1,2, \ldots\}$ the set of natural numbers, $\mathbb{R}$ the set of real numbers, and $\mathbb{C}$ the set of complex numbers.

If $M$ is an infinite subset of $\mathbb{N}$, we denote by $[M]$ the set of all infinite subsets of $M$, considering them as strictly increasing sequences, and if $s$ is a non-empty finite subset of $\mathbb{N}$, we set 
$[s, M]=\{s \cup L \in[\mathbb{N}]: L \in[M]$ and $\max s<\min L\} \quad$ if $s \neq \emptyset$, $[\emptyset, M]=[M]$.

Universal series. Fix a sequence $\left(X_{k}, \varrho_{k}\right)_{k \geq 1}$ of separable, metrizable topological vector spaces over the field $\mathbb{K}=\mathbb{R}$ or $\mathbb{C}$, equipped with translation-invariant metrics $\varrho_{k}$, and fix sequences $\left(x_{n}^{k}\right)_{n=0}^{\infty} \subseteq X_{k}$ for every $k \geq 1$.

Definition 1.1. A sequence $\left(\alpha_{n}\right)_{n=0}^{\infty} \in \mathbb{K}^{\mathbb{N}}$ belongs to the class $\mathcal{U}$ if for every $k \geq 1$ the set $\left\{\sum_{j=0}^{n} \alpha_{j} x_{j}^{k}: n \in \mathbb{N}\right\}$ is dense in $X_{k}$.

If $\mathcal{U} \neq \emptyset$, each $\left(\alpha_{n}\right)_{n=0}^{\infty} \in \mathcal{U}$ is said to generate an unrestricted universal series.

Of special interest is the case where some elements of the class $\mathcal{U}$ satisfy certain restrictions. The restricted universal series are defined as follows:

We fix a vector subspace $A$ of $\mathbb{K}^{\mathbb{N}}$, and assume that $A$ is equipped with a complete metrizable vector space topology, induced by a translationinvariant metric $d$, such that

(i) the projections $p_{m}: A \rightarrow \mathbb{K},\left(\alpha_{n}\right)_{n=0}^{\infty} \mapsto a_{m}$, are continuous for every $m \in \mathbb{N}$,

(ii) $c_{00}=\left\{\left(\alpha_{n}\right)_{n=0}^{\infty} \in \mathbb{K}^{\mathbb{N}}:\left\{n \in \mathbb{N}: \alpha_{n} \neq 0\right\}\right.$ is finite $\}$ is a dense subset of $A$.

Definition 1.2. A sequence $\alpha=\left(\alpha_{n}\right)_{n=0}^{\infty} \in A$ belongs to the class $\mathcal{U}_{A}$ if, for every $k \geq 1$ and every $x \in X_{k}$, there exists a sequence $\left(\lambda_{n}\right)_{n=1}^{\infty} \subseteq \mathbb{N}$ such that

$$
\varrho_{k}\left(\sum_{j=0}^{\lambda_{n}} \alpha_{j} x_{j}^{k}, x\right) \rightarrow 0 \quad \text { and } \quad d\left(\sum_{j=0}^{\lambda_{n}} \alpha_{j} e_{j}, \alpha\right) \rightarrow 0 \quad \text { as } n \rightarrow \infty,
$$

where $e_{0}=(1,0,0, \ldots), e_{1}=(0,1,0, \ldots), e_{2}=(0,0,1,0, \ldots), \ldots$

REMARK 1.3. In Definition 1.2, we can assume, without loss of generality, that $\lambda_{n}<\lambda_{n+1}$ for all $n \in \mathbb{N}$.

Definition 1.4. For every $k \geq 1$, let $T_{n}^{k}: A \rightarrow X_{k}, n \in \mathbb{N}$, be a sequence of continuous functions, and let $\alpha=\left(\alpha_{n}\right)_{n=0}^{\infty} \in A$. By definition:

(1) $\alpha \in \mathcal{F}_{1}$ if, for every $k \geq 1$ and every $x \in X_{k}$, there exists a sequence $\left(\lambda_{n}\right)_{n=1}^{\infty} \subseteq \mathbb{N}$ such that

$$
\varrho_{k}\left(T_{\lambda_{n}}^{k}(\alpha), x\right) \rightarrow 0 \quad \text { as } n \rightarrow \infty .
$$

(2) $\alpha \in \mathcal{F}_{2}$ if, for every $k \geq 1$ and every $x \in X_{k}$, there exists a sequence $\left(\lambda_{n}\right)_{n=1}^{\infty} \subseteq \mathbb{N}$ such that

$$
\varrho_{k}\left(T_{\lambda_{n}}^{k}(\alpha), x\right) \rightarrow 0 \quad \text { and } \quad d\left(\sum_{j=0}^{\lambda_{n}} \alpha_{j} e_{j}, \alpha\right) \rightarrow 0 \quad \text { as } n \rightarrow \infty .
$$


(3) $\alpha \in \mathcal{F}_{3}$ if, for every $k \geq 1$ and every $x \in X_{k}$, there exists a sequence $\left(\lambda_{n}\right)_{n=1}^{\infty} \subseteq \mathbb{N}$ such that

$$
\varrho_{k}\left(T_{\lambda_{n}}^{k}(\alpha), x\right) \rightarrow 0, \quad d\left(\sum_{j=0}^{\lambda_{n}} \alpha_{j} e_{j}, \alpha\right) \rightarrow 0, \quad \varrho_{k}\left(\sum_{j=0}^{\lambda_{n}} \alpha_{j} x_{j}^{k}, x\right) \rightarrow 0
$$

as $n \rightarrow \infty$.

We refer the reader to $[\mathrm{G}-\mathrm{E}],[\mathrm{NP}]$ and $[\mathrm{BGNP}]$ for definitions, results, and interesting examples on the classes $\mathcal{U}, \mathcal{U}_{A}$, and also for the proof of the following Proposition 1.5. A sketch of the proof is included for completeness.

Proposition 1.5. Under the previous assumptions, the classes $\mathcal{U} \cap A$, $\mathcal{U}_{A}, \mathcal{F}_{1}, \mathcal{F}_{2}$ and $\mathcal{F}_{3}$ are $G_{\delta}$ subsets of the space $A$. In particular, for $A=\mathbb{K}^{\mathbb{N}}$, the class $\mathcal{U}$ is a $G_{\delta}$ subset of the space $\mathbb{K}^{\mathbb{N}}$ with the product topology.

Sketch of the proof. Let $\left(y_{n}^{k}\right)_{n=0}^{\infty}$ be a dense sequence in $X_{k}$ for every $k \geq 1$. For $n, l, r, k \in \mathbb{N}$ with $r, k \geq 1$, consider the sets

$$
\begin{gathered}
E(n, l, r, k)=\left\{\alpha=\left(\alpha_{n}\right)_{n=0}^{\infty} \in A: \varrho_{k}\left(\sum_{j=0}^{n} \alpha_{j} x_{j}^{k}, y_{l}^{k}\right)<1 / r\right\}, \\
D(n, r)=\left\{\alpha=\left(\alpha_{n}\right)_{n=0}^{\infty} \in A: d\left(\sum_{j=0}^{n} \alpha_{j} e_{j}, \alpha\right)<1 / r\right\}, \\
C(n, l, r, k)=\left\{\alpha=\left(\alpha_{n}\right)_{n=0}^{\infty} \in A: \varrho_{k}\left(T_{n}^{k}(\alpha), y_{l}^{k}\right)<1 / r\right\} .
\end{gathered}
$$

Under our assumptions on $A$, the sets $E(n, l, r, k), D(n, r), C(n, l, r, k)$ are open in $A$, and

$$
\begin{aligned}
\mathcal{U} \cap A & =\bigcap_{l, r, k} \bigcup_{n=0}^{\infty} E(n, l, r, k) \\
\mathcal{U}_{A} & =\bigcap_{l, r, k} \bigcup_{n=0}^{\infty}(E(n, l, r, k) \cap D(n, r)), \\
\mathcal{F}_{1} & =\bigcap_{l, r, k} \bigcup_{n=0}^{\infty} C(n, l, r, k), \\
\mathcal{F}_{2} & =\bigcap_{l, r, k} \bigcup_{n=0}^{\infty}(C(n, l, r, k) \cap D(n, r)), \\
\mathcal{F}_{3} & =\bigcap_{l, r, k} \bigcup_{n=0}^{\infty}(C(n, l, r, k) \cap D(n, r) \cap E(n, l, r, k)) .
\end{aligned}
$$

Borel and analytic sets. Let $X, Y$ be Polish spaces (i.e. topological Hausdorff spaces, each homeomorphic to a complete, metric, separable space), 
$f: X \rightarrow Y$ a continuous function, and $B$ a Borel subset of $X$. The image $C=f(B)$ is not always a Borel subset of $Y$, in fact all such subsets $C$ of $Y$ constitute the class of analytic subsets of $Y$. Moreover, the analytic subsets of $Y$ are characterized as the results of the Suslin operation on the class of closed subsets of $Y$ (see [Ke]).

We will use the following highly non-trivial result about Borel sets. A proof can be found in Theorem 15.1 of [Ke].

Theorem 1.6 (Lusin, Suslin). Let $X, Y$ be Polish spaces and $f: X \rightarrow Y$ a continuous function. If $B$ is a Borel subset of $X$, and $f$ restricted to $B$ is one-to-one, then $f(B)$ is a Borel subset of $Y$.

Infinitary combinatorics. Galvin and Prikry [GP] proved the following fundamental combinatorial result for infinite sequences of natural numbers.

THEOREM 1.7. Let $\mathcal{R}$ be a family of infinite subsets of the space $[\mathbb{N}]$, endowed with the relative topology in the space $\mathbb{N}^{\mathbb{N}}$ with the product topology. Assume that $\mathcal{R}$ is a Borel subset of $[\mathbb{N}]$, and let $s$ be a finite subset of $\mathbb{N}$ and $M$ an infinite subset of $\mathbb{N}$. Then there exists $L \in[M]$ such that

$$
\text { either }[s, L] \subseteq \mathcal{R}, \quad \text { or } \quad[s, L] \subseteq[\mathbb{N}] \backslash \mathcal{R} .
$$

Silver $[\mathrm{Si}]$ proved an analogous result in the more general case where $\mathcal{R}$ is an analytic subset of $[\mathbb{N}]$, and Ellentuck $[\mathrm{E}]$ formulated a still more general result.

2. The dichotomy principle. Combining the Galvin and Prikry combinatorial result of Theorem 1.7 with the result of Lusin and Suslin about Borel sets (Theorem 1.6), we can prove a general dichotomy for classes of universal series.

TheOREM 2.1. Let $A$ be a vector subspace of $\mathbb{K}^{\mathbb{N}}(\mathbb{K}=\mathbb{R}$ or $\mathbb{C})$ which is equipped with a complete metrizable vector space topology, induced by a translation-invariant metric $d$, and satisfies properties (i) and (ii) of Section 1 , and let $\mathcal{G}$ be a Borel subset of $A$. Then for every sequence $\left(\alpha_{n}\right)_{n=0}^{\infty}$ in $\mathbb{K}$, every finite subset $s$ of $\mathbb{N}$ and every infinite subset $M$ of $\mathbb{N}$ there exists an infinite subset $L$ of $M$ such that either

- all subsequences $\left(\alpha_{i_{n}}\right)_{n=0}^{\infty}$ of $\left(\alpha_{n}\right)_{n=0}^{\infty}$ with $\left(i_{n}\right)_{n=1}^{\infty} \in[s, L]$ belong to $\mathcal{G}$, or

- all subsequences $\left(\alpha_{i_{n}}\right)_{n=0}^{\infty}$ of $\left(\alpha_{n}\right)_{n=0}^{\infty}$ with $\left(i_{n}\right)_{n=0}^{\infty} \in[s, L]$ belong to $\mathbb{K}^{\mathbb{N}} \backslash \mathcal{G}$.

In particular, the conclusion holds if we replace the class $\mathcal{G}$ by each of the classes $\mathcal{U} \cap A, \mathcal{U}_{A}, \mathcal{F}_{1}, \mathcal{F}_{2}$ and $\mathcal{F}_{3}$ (defined in Section 1). For $A=\mathbb{K}^{\mathbb{N}}$ the conclusion holds for $\mathcal{G}=\mathcal{U}=\mathcal{U} \cap A$ as well. 
Proof. We set $\mathcal{R}=\left\{I=\left(i_{n}\right)_{n=0}^{\infty} \in[\mathbb{N}]:\left(\alpha_{i_{n}}\right)_{n=0}^{\infty} \in \mathcal{G}\right\}$, and we claim that $\mathcal{R}$ is a Borel subset of $[\mathbb{N}]$, endowed with the relative product topology of $\mathbb{N}^{\mathbb{N}}$.

Indeed, the function $f:[\mathbb{N}] \rightarrow \mathbb{K}^{\mathbb{N}}$ with $f\left(\left(i_{n}\right)_{n=0}^{\infty}\right)=\left(\alpha_{i_{n}}\right)_{n=0}^{\infty}$ is continuous if $\mathbb{K}^{\mathbb{N}}$ is endowed with the product topology. Notice that $\mathcal{R}=f^{-1}(\mathcal{G})$.

The set $\mathcal{G}$ is a Borel subset of $\mathbb{K}^{\mathbb{N}}$. Indeed, the identity function $g$ : $A \rightarrow \mathbb{K}^{\mathbb{N}}$ with $g(\alpha)=\alpha$ is continuous, since by property (i), the projections $p_{m}: A \rightarrow \mathbb{K},\left(\alpha_{n}\right)_{n=0}^{\infty} \rightarrow a_{m}$, are continuous for every $m \in \mathbb{N}$. Certainly $\mathbb{K}^{\mathbb{N}}$ is a Polish space, and also $A$ is a Polish space, since it is a complete metrizable space with property (ii). Hence $\mathcal{G}=g(\mathcal{G})$ is a Borel subset of $\mathbb{K}^{\mathbb{N}}$, according to the Lusin-Suslin Theorem 1.6.

Since the function $f:[\mathbb{N}] \rightarrow \mathbb{K}^{\mathbb{N}}$ is continuous, the set $\mathcal{R}=f^{-1}(\mathcal{G})$ is a Borel subset of $[\mathbb{N}]$.

Now, we can apply the Galvin-Prikry Theorem 1.7 for the family $\mathcal{R}$. It follows that there exists $L \in[M]$ such that either $[s, L] \subseteq \mathcal{R}$, or $[s, L] \subseteq$ $[\mathbb{N}] \backslash \mathcal{R}$. Hence, the subsequences $\left(\alpha_{i_{n}}\right)_{n=0}^{\infty}$ of $\left(\alpha_{n}\right)_{n=0}^{\infty}$ with $\left(i_{n}\right)_{n=0}^{\infty} \in[s, L]$ either all belong to the class $\mathcal{G}$, in case $[s, L] \subseteq \mathcal{R}$, or all belong to $\mathbb{K}^{\mathbb{N}} \backslash \mathcal{G}$, in case $[s, L] \subseteq[\mathbb{N}] \backslash \mathcal{R}$.

In particular, taking for $\mathcal{G}$ each of the classes $\mathcal{U} \cap A, \mathcal{U}_{A}, \mathcal{F}_{1}, \mathcal{F}_{2}$ and $\mathcal{F}_{3}$, since all these classes, according to Proposition 1.5, are $G_{\delta}$ subsets of $A$, we have the conclusion for each of these classes. In particular, applying the arguments for $\mathcal{G}=\mathcal{U}$ and $A=\mathbb{K}^{\mathbb{N}}$ with the product topology, we have the conclusion for the class $\mathcal{U}$.

Corollary 2.2. Let $\left(X_{k}\right)_{k \geq 1}$ be a sequence of separable, metrizable topological vector spaces over the field $\mathbb{K}=\mathbb{R}$ or $\mathbb{C}$, equipped with translationinvariant metrics $\varrho_{k}$ for every $k \geq 1$, let $A$ be a vector subspace of $\mathbb{K}^{\mathbb{N}}$, equipped with a complete metrizable vector space topology, induced by a translation-invariant metric d, and satisfying properties (i) and (ii) of Section 1 , and let sequences $\left(x_{n}^{k}\right)_{n=0}^{\infty}$ be in $X_{k}$ for every $k \geq 1$. Then, for every sequence $\left(\alpha_{n}\right)_{n=0}^{\infty}$ in $\mathbb{K}$ and $\mathcal{G}$ equal to one of the classes $\mathcal{U} \cap A, \mathcal{U}_{A}, \mathcal{F}_{1}, \mathcal{F}_{2}$ or $\mathcal{F}_{3}$, there exists a subsequence $\left(\alpha_{l_{n}}\right)_{n=0}^{\infty}$ of $\left(\alpha_{n}\right)_{n=0}^{\infty}$ such that either

- all the subsequences of $\left(\alpha_{l_{n}}\right)_{n=0}^{\infty}$ belong to $\mathcal{G}$, or

- all the subsequences of $\left(\alpha_{l_{n}}\right)_{n=0}^{\infty}$ belong to $\mathbb{K}^{\mathbb{N}} \backslash \mathcal{G}$.

Proof. We apply Theorem 2.1 in the case $s=\emptyset$.

Theorem 2.1 can be stated in a more general form, assuming the partition family to be analytic instead of Borel.

THEOREM 2.3. Let $A$ be a vector subspace of $\mathbb{K}^{\mathbb{N}}$ (where $\mathbb{K}=\mathbb{R}$ or $\mathbb{C}$ ), equipped with a complete metrizable vector space topology, induced by a translation-invariant metric $d$ and satisfying properties (i) and (ii) of Section 1 , and let $\mathcal{D}$ be an analytic subset of $A$. Then for every sequence $\left(\alpha_{n}\right)_{n=0}^{\infty}$ 
in $\mathbb{K}$, every finite subset $s$ of $\mathbb{N}$, and every infinite subset $M$ of $\mathbb{N}$, there exists an infinite subset $L$ of $M$ such that either

- all subsequences $\left(\alpha_{i_{n}}\right)_{n=0}^{\infty}$ of $\left(\alpha_{n}\right)_{n=0}^{\infty}$ with $\left(i_{n}\right)_{n=0}^{\infty} \in[s, L]$ belong to $\mathcal{D}$, or

- all subsequences $\left(\alpha_{i_{n}}\right)_{n=0}^{\infty}$ of $\left(\alpha_{n}\right)_{n=0}^{\infty}$ with $\left(i_{n}\right)_{n=0}^{\infty} \in[s, L]$ belong to $\mathbb{K}^{\mathbb{N}} \backslash \mathcal{D}$.

Proof. We note that the family $\mathcal{R}=\left\{I=\left(i_{n}\right)_{n=0}^{\infty} \in[\mathbb{N}]:\left(\alpha_{i_{n}}\right)_{n=0}^{\infty} \in \mathcal{D}\right\}$ is an analytic subset of $[\mathbb{N}]$ endowed with the relative product topology of $\mathbb{N}^{\mathbb{N}}$.

Indeed, the set $\mathcal{D}=g(\mathcal{D})$ is an analytic subset of $\mathbb{K}^{\mathbb{N}}$ with the product topology, as the identity function $g: A \rightarrow \mathbb{K}^{\mathbb{N}}$ is continuous and $\mathcal{D}$ is an analytic subset of the Polish space $A$. Since the function $f:[\mathbb{N}] \rightarrow \mathbb{K}^{\mathbb{N}}$ with $f\left(\left(i_{n}\right)_{n=0}^{\infty}\right)=\left(\alpha_{i_{n}}\right)_{n=0}^{\infty}$ is continuous, we see that $\mathcal{R}=f^{-1}(\mathcal{D})$ is an analytic subset of $[\mathbb{N}]$. This last conclusion can be proved easily by using the characterization of the analytic subsets of a Polish space $X$, as those that result from the Suslin operation on the class of closed subsets of $X$.

Now, we apply the result by Silver [Si], or by Ellentuck [E] (in place of the Galvin-Prikry Theorem 1.7, replacing the Borel partition by a partition determined by an analytic set). So, there exists $L \in[M]$ such that either $[s, L] \subseteq \mathcal{D}$, or $[s, L] \subseteq[\mathbb{N}] \backslash \mathcal{D}$. This finishes the proof.

REMARK 2.4. At present, we have no use for the more general results in Theorem 2.1, on partitions determined by an analytic set, but it appears that the full force of the Galvin-Prikry theorem for Borel partitions is employed in the proof of our main result 2.1. If this is indeed the case, then Theorem 2.1 is the only "natural theorem" that uses the full strength of the Galvin-Prikry partition theorem. (Cf. the relevant remark after Theorem 5.7 in Gowers's survey paper [G].)

REMARK 2.5. For a fixed strictly increasing sequence $\mu=\left(\mu_{n}\right)_{n=1}^{\infty}$ in $\mathbb{N}$, we can define the classes $\mathcal{U}^{\mu}, \mathcal{U}^{\mu} \cap A, \mathcal{U}_{A}^{\mu}, \mathcal{F}_{1}^{\mu}, \mathcal{F}_{2}^{\mu}, \mathcal{F}_{3}^{\mu}$ analogously to $\mathcal{U}, \mathcal{U} \cap A$, $\mathcal{U}_{A}, \mathcal{F}_{1}, \mathcal{F}_{2}, \mathcal{F}_{3}$, with the only difference that the sequence $\left(\lambda_{n}\right)_{n=1}^{\infty} \subseteq \mathbb{N}$ in Definitions 1.2 and 1.4 has to be a subsequence of $\mu$. All the results which we proved for $\mathcal{U}, \mathcal{U} \cap A, \mathcal{U}_{A}, \mathcal{F}_{1}, \mathcal{F}_{2}, \mathcal{F}_{3}$ also hold for $\mathcal{U}^{\mu}, \mathcal{U}^{\mu} \cap A, \mathcal{U}_{A}^{\mu}, \mathcal{F}_{1}^{\mu}$, $\mathcal{F}_{2}^{\mu}$ and $\mathcal{F}_{3}^{\mu}$.

3. Particular cases and examples. In this section we examine some particular cases of universality in relation with Corollary 2.2; in addition, concrete examples are used to show that the classes $\mathcal{U}_{A}$ and $\mathbb{K}^{\mathbb{N}} \backslash \mathcal{U}_{A}$, as well as $\mathcal{U} \cap A$ and $\mathbb{K}^{\mathbb{N}} \backslash(\mathcal{U} \cap A)$, are not always hereditary (where a class $\mathcal{F}$ in $\mathbb{K}^{\mathbb{N}}$ is hereditary if every subsequence of any sequence in $\mathcal{F}$ belongs to $\mathcal{F}$ ). 
Fix a separable Banach space $X$ and a sequence $\left(x_{n}\right)_{n=0}^{\infty}$ in $X$. We consider the particular case of Definition 1.1, where $X_{k}=X$ and $x_{n}^{k}=x_{n}$ for all $k=1,2, \ldots$ In this case we prove the following.

Proposition 3.1. Let $X$ be a Banach space, and $\left(x_{j}\right)_{j=0}^{\infty} \subseteq X$ with $x_{j} \neq 0$ for every $j \in \mathbb{N}$. Set $A=c_{0}$ or $A=l^{p}$ with $0<p<\infty$, and let $\left(\alpha_{n}\right)_{n=0}^{\infty} \in A$. Then there exists a subsequence $\left(\alpha_{l_{n}}\right)_{n=0}^{\infty}$ of $\left(\alpha_{n}\right)_{n=0}^{\infty}$ with no subsequences in $\mathcal{U} \cap A=\mathcal{U}_{A}$.

Proof. Let $\left(\alpha_{l_{n}}\right)_{n=0}^{\infty}$ be a subsequence of $\left(\alpha_{n}\right)_{n=0}^{\infty}$ with

$$
\left|\alpha_{l_{n}}\right| \leq \frac{1}{(n+1)^{2} \varrho\left(x_{j}, 0\right)} \quad \text { for every } n \in \mathbb{N} \text { and } j=0, \ldots, n .
$$

For every subsequence $\left(\alpha_{k_{l_{n}}}\right)_{n=0}^{\infty}$ of $\left(\alpha_{l_{n}}\right)_{n=0}^{\infty}$ we have $k_{l_{n}} \geq n$ for every $n \in \mathbb{N}$, and consequently $\left\|\alpha_{k_{n}} x_{n}\right\| \leq 1 /\left(l_{n}+1\right)^{2}$, which implies that the series $\sum_{n=0}^{\infty} \alpha_{k_{l_{n}}} x_{n}$ converges in $X$. Therefore $\left(\alpha_{k_{l_{n}}}\right)_{n=0}^{\infty}$ does not belong to $\mathcal{U} \cap A=\mathcal{U}_{A}$.

Proposition 3.2. Let $1<R<\infty$, set $D(0, R)=\{z \in \mathbb{C}:|z|<R\}$ and

$$
A=\left\{\left(\alpha_{n}\right)_{n=0}^{\infty}: \sum_{n=0}^{\infty} \alpha_{n} z^{n} \text { converges in } D(0, R)\right\} \text {, }
$$

and endow $A$ with the metric $d$ which is the image, under the bijective map $g: A \rightarrow H(D(0, R))$ with $g\left(\left(\alpha_{n}\right)_{n=0}^{\infty}\right)=\sum_{n=0}^{\infty} \alpha_{n} z^{n}$, of the standard metric $\tilde{d}$ on $H(D(0, R))$, compatible with the topology of uniform convergence on compact subsets of $D(0, R)$. Also assume ( $f$. $[\mathrm{N}],[\mathrm{NP}]$ and [BGNP]) that there exists a sequence $\left(X_{k}, \varrho_{k}\right)_{k \geq 1}$ of separable, metrizable topological vector spaces over $\mathbb{C}$, and sequences $\left(x_{n}^{k}\right)_{n=0}^{\infty} \subseteq X_{k}$ for every $k \geq 1$, such that $\mathcal{U} \cap A=\mathcal{U}_{A}$ is the class of universal Taylor series, namely the class of all sequences $\left(\alpha_{n}\right)_{n=0}^{\infty} \in A$ for which for every compact set $K \subseteq \mathbb{C}$ with $K \cap D(0, R)=\emptyset$ and $\mathbb{C} \backslash K$ connected, and for every function $h: K \rightarrow \mathbb{C}$, continuous on $K$ and holomorphic in the interior of $K$, there exists a sequence $\left(\lambda_{n}\right)_{n=1}^{\infty} \subseteq \mathbb{N}$ with $\sum_{j=0}^{\lambda_{n}} \alpha_{j} z^{j} \stackrel{n}{\rightarrow} h(z)$ uniformly on $K$. Then, for every sequence $\left(\alpha_{n}\right)_{n=0}^{\infty} \in A$, there exists a subsequence $\left(\alpha_{l_{n}}\right)_{n=0}^{\infty}$ of $\left(\alpha_{n}\right)_{n=0}^{\infty}$ with no subsequences in $\mathcal{U} \cap A=\mathcal{U}_{A}$.

Proof. Set $K=\{R\}$. We can have $X_{1}=\mathbb{C}=\{f: K \rightarrow \mathbb{C}\}, \varrho_{1}$ the usual metric on $\mathbb{C}$ and $x_{j}^{1}=\left.z^{j}\right|_{K}$ for every $j \in \mathbb{N}$. Since $\left(\alpha_{n}\right)_{n=0}^{\infty} \in A$ and $R>1$, it is easily seen that $\alpha_{n} \rightarrow 0$, hence we can choose $\left(l_{n}\right)_{n=0}^{\infty} \subseteq \mathbb{N}$ with

$$
\left|\alpha_{l_{n}}\right| \leq \frac{1}{(n+1)^{2} R^{j}}=\frac{1}{(n+1)^{2} \varrho\left(x_{j}^{1}, 0\right)} \quad \text { for every } n \in \mathbb{N} \text { and } j=0, \ldots, n \text {. }
$$

The rest of the proof is similar to that of Proposition 3.1. 
Proposition 3.3. Let $X$ be a separable Banach space and $\left(x_{j}\right)_{j=0}^{\infty} \subseteq X$ with $x_{j} \neq 0$ for every $j \in \mathbb{N}$. Then for every sequence $\left(\alpha_{n}\right)_{n=0}^{\infty} \in \mathbb{K}^{\mathbb{N}}$, we have the following cases:

(a) if $\left(\alpha_{n}\right)_{n=0}^{\infty}$ has zero or infinity as accumulation points, then it has a subsequence with no subsequences in $\mathcal{U}$;

(b) if $\left(\alpha_{n}\right)_{n=0}^{\infty}$ has a subsequence converging to some $c \in \mathbb{K}$ with $c \neq 0$, and $(1,1, \ldots) \in \mathcal{U}$, then it has a subsequence with all subsequences in $\mathcal{U}$

(c) if $\left(\alpha_{n}\right)_{n=0}^{\infty}$ has a subsequence converging to some $c \in \mathbb{K}$ with $c \neq 0$, and $(1,1, \ldots) \notin \mathcal{U}$, then it has a subsequence with no subsequences in $\mathcal{U}$.

Proof. If $\left(\alpha_{n}\right)_{n=0}^{\infty}$ has a subsequence converging to zero, then, as in the proof of Proposition 3.1, we can construct a subsequence $\left(\alpha_{l_{n}}\right)_{n=0}^{\infty}$ with no subsequences in $\mathcal{U}$.

Let $\left(\alpha_{n}\right)_{n=0}^{\infty}$ have a subsequence converging to some $c \in \mathbb{K}$ with $c \neq 0$. Then we can find a subsequence $\left(\alpha_{l_{n}}\right)_{n=0}^{\infty}$ with

$$
\left|\alpha_{l_{n}}-c\right| \leq \frac{1}{(n+1)^{2} \varrho\left(x_{j}, 0\right)} \quad \text { for every } n \in \mathbb{N} \text { and } j=0, \ldots, n \text {. }
$$

Hence, $\sum_{n=0}^{\infty}\left(\alpha_{k_{l_{n}}}-c\right) x_{n}$ converges in $X$ for every subsequence $\left(\alpha_{k_{l_{n}}}\right)_{n=0}^{\infty}$ of $\left(\alpha_{l_{n}}\right)_{n=0}^{\infty}$. Therefore, in case $(c, c, \ldots) \in \mathcal{U}$ all the subsequences of $\left(\alpha_{l_{n}}\right)_{n=0}^{\infty}$ belong to $\mathcal{U}$, and in case $(c, c, \ldots) \notin \mathcal{U}$ no one does. But $(c, c, \ldots) \in \mathcal{U}$ if and only if $(1,1, \ldots) \in \mathcal{U}$.

Finally, it remains to examine the case where $\left(\alpha_{n}\right)_{n=0}^{\infty}$ has a subsequence converging to infinity. Then we can find a subsequence $\left(\alpha_{l_{n}}\right)_{n=0}^{\infty}$ such that for all $n \in\{1,2, \ldots\}$ and all $F \subseteq \mathbb{N}$ with $F \subseteq\{0, \ldots, n-1\}$ we have

$$
\left\|\left(\sum_{j \in F} \alpha_{l_{j}} x_{j}\right)+\alpha_{l_{n}} x_{n}\right\| \geq 1
$$

by choosing $l_{n} \in \mathbb{N}$ with $\left\|\alpha_{l_{n}} x_{n}\right\| \geq 1+\left\|\sum_{j \in F} \alpha_{l_{j}} x_{j}\right\|$ for every such $F$. Hence, for every subsequence $\left(\alpha_{k_{l_{n}}}\right)_{n=0}^{\infty}$ of $\left(\alpha_{l_{n}}\right)_{n=0}^{\infty}$ the set $\left\{\sum_{n=0}^{N} \alpha_{k_{l_{n}}} x_{n}\right.$ : $N \in \mathbb{N}\}$ avoids the open set $\{x \in X:\|x\|<1\}$, so it is not dense in $X$. Thus $\left(\alpha_{k_{l_{n}}}\right)_{n=0}^{\infty} \notin \mathcal{U}$.

This completes the proof.

The following theorem extends Corollary 2.2 to the class $\mathcal{U} \cap A$ in the particular case where $A$ is a hereditary family, thus providing a criterion for all the subsequences to be in $\mathcal{U} \cap A$ or in its complement.

Theorem 3.4. Let $X$ be a separable Banach space, $\left(x_{j}\right)_{j=0}^{\infty} \subseteq X$ with $x_{j} \neq 0$ for every $j \in \mathbb{N}$, and $A$ a hereditary vector subspace of $\mathbb{K}^{\mathbb{N}}$ (with $\mathbb{K}=\mathbb{R}$ or $\mathbb{C})$, equipped with a complete metrizable vector space topology, 
induced by a translation-invariant metric d, and satisfying properties (i) and (ii) of Section 1. Then for every sequence $\left(\alpha_{n}\right)_{n=0}^{\infty}$ in $\mathbb{K}$ we have the following cases:

(a) if $(1,1, \ldots) \in \mathcal{U}$ and $\left(\alpha_{n}\right)_{n=0}^{\infty}$ has a subsequence in $A$ converging to some $c \in \mathbb{K}$ with $c \neq 0$, then $\left(\alpha_{n}\right)_{n=0}^{\infty}$ has a subsequence with all subsequences in $\mathcal{U} \cap A$;

(b) if either $(1,1, \ldots) \notin \mathcal{U}$, or $(1,1, \ldots) \in \mathcal{U}$ and $\left(\alpha_{n}\right)_{n=0}^{\infty}$ has no subsequence in $A$ converging to some $c \in \mathbb{K}$ with $c \neq 0$, then $\left(\alpha_{n}\right)_{n=0}^{\infty}$ has a subsequence with no subsequences in $\mathcal{U} \cap A$.

Moreover, if $\left(\alpha_{n}\right)_{n=0}^{\infty}$ has a subsequence converging to zero or to infinity, then $\left(\alpha_{n}\right)_{n=0}^{\infty}$ has a subsequence with no subsequences in $\mathcal{U} \cap A$.

Proof. If $(1,1, \ldots) \notin \mathcal{U}$, then, according to Proposition $3.3,\left(\alpha_{n}\right)_{n=0}^{\infty}$ has a subsequence with no subsequences in $\mathcal{U}$ and therefore none in $\mathcal{U} \cap A$.

If $(1,1, \ldots) \in \mathcal{U}$ and $\left(\alpha_{n}\right)_{n=0}^{\infty}$ has a subsequence converging to zero or to infinity, then, according to Proposition $3.3,\left(\alpha_{n}\right)_{n=0}^{\infty}$ has a subsequence with no subsequences in $\mathcal{U}$ and therefore none in $\mathcal{U} \cap A$.

If $(1,1, \ldots) \in \mathcal{U}$ and $\left(\alpha_{n}\right)_{n=0}^{\infty}$ has all its accumulation points in $\mathbb{K} \backslash\{0\}$, then, according to Proposition 3.3, it has a subsequence $\left(\alpha_{l_{n}}\right)_{n=0}^{\infty}$ with all subsequences in $\mathcal{U}$. Now, if one subsequence $\left(\alpha_{l_{k_{n}}}\right)_{n=0}^{\infty}$ belongs to the class $A$, then all its subsequences belong to $\mathcal{U} \cap A$, since $A$ is hereditary, and if no subsequence of $\left(\alpha_{l_{n}}\right)_{n=0}^{\infty}$ belongs to $A$, then none belongs to $\mathcal{U} \cap A$.

REMARK 3.5. (1) In order to give an example of a sequence in $\mathcal{U} \cap A$ with a subsequence not in $\mathcal{U}$, we apply Proposition 3.1 for $A=c_{0}$ and we notice that $\mathcal{U} \cap c_{0} \neq \emptyset$ in the case of universal trigonometric series in the sense of Men'shov ([KN], [BGNP]), as well as in the case of universal Taylor series in the open unit disk in the sense of Luh ([L]) and Chui-Parnes ([CP]), where the universal approximation is not required on the boundary ([KKN], $[\mathrm{MN}]$, [BGNP]).

For another example, start with a sequence $\left(x_{n}\right)_{n=0}^{\infty}$ in a Banach space $X$ such that the set $\left\{\sum_{n=0}^{N} x_{2 n}: N \in \mathbb{N}\right\}$ is dense in $X$. Then the sequence $(1,0,1,0, \ldots)$ belongs to $\mathcal{U}$ and obviously its subsequence $(0,0,0,0, \ldots)$ does not.

(2) In order to give an example of a sequence not in $\mathcal{U}$ with a subsequence in $\mathcal{U}$, we start with a sequence $\left(x_{n}\right)_{n=0}^{\infty}$ dense in a Banach space $X$ and we consider the sequence $\left(y_{n}\right)_{n=0}^{\infty} \subseteq X$ where $y_{0}=x_{0}$ and $y_{2 n-1}=y_{2 n}=x_{n}$ for every $n \geq 1$, which is also dense in $X$. Fix $\left(z_{n}\right)_{n=0}^{\infty} \subseteq X$, where $z_{0}=y_{0}=x_{0}$ and $z_{n}=y_{n}-y_{n-1}$ for every $n \geq 1$. Then $z_{2 n}=0$ and $z_{2 n-1}=y_{n}-y_{n-1}$ for every $n \geq 1$.

The sequence $\left(\alpha_{n}\right)_{n=0}^{\infty} \subseteq \mathbb{R}$ with $\alpha_{2 n+1}=0$ and $\alpha_{2 n}=1$ for every $n \in \mathbb{N}$ does not belong to $\mathcal{U}$. Indeed, for every $N \in \mathbb{N}$ we have $\sum_{n=0}^{N} \alpha_{n} z_{n}=y_{0}$. On 
the other hand, the subsequence $\left(\alpha_{2 n}\right)_{n=0}^{\infty}$ belongs to $\mathcal{U}$, since $\sum_{n=0}^{2 N} \alpha_{2 n} z_{n}=$ $\sum_{n=0}^{2 N} z_{n}=y_{N}$ for every $N \in \mathbb{N}$ and $\left(y_{N}\right)_{N=0}^{\infty} \subseteq X$ is dense in $X$.

Acknowledgments. We wish to thank the Department of Mathematics and Statistics of University of Cyprus for the warm hospitality extended during our visit in the spring semester of 2007 , and also A. Tsarpalias for helpful discussions. Both authors acknowledge partial support by the Kapodistrias research grant of Athens University.

\section{References}

[BGNP] F. Bayart, K.-G. Grosse-Erdmann, V. Nestoridis and C. Papadimitropoulos, Abstract theory of universal series and applications, Proc. London Math. Soc. 96 (2008), 417-463.

[CP] C. Chui and M. N. Parnes, Approximation by overconvergence of power series, J. Math. Anal. Appl. 36 (1971), 693-696.

[E] E. Ellentuck, A new proof that analytic sets are Ramsey, J. Symbolic Logic 39 (1974), 163-164.

[F] V. Farmaki, Ramsey and Nash-Williams combinatorics via Schreier families, arXiv:math.FA/0404014 v.1, (2004), electronic prepublication.

[FN1] V. Farmaki and S. Negrepontis, Block combinatorics, Trans. Amer. Math. Soc. 358 (2006), 2759-2779.

[FN2] -, 一, Schreier sets in Ramsey theory, ibid. 360 (2008), 849-880.

[GP] F. Galvin and K. Prikry, Borel sets and Ramsey's theorem, J. Symbolic Logic 38 (1973), 193-198.

[G] W. T. Gowers, Ramsey methods in Banach spaces, in: Handbook of the Geometry of Banach Spaces 2, W. B. Johnson and J. Lindenstrauss (eds.), 2003, Elsevier, 1071-1097.

[G-E] K.-G. Grosse-Erdmann, Universal families and hypercyclic operators, Bull. Amer. Math. Soc. 36 (1999), 345-381.

[K] J.-P. Kahane, Baire's category theorem and trigonometric series, J. Anal. Math. 80 (2000), 143-182.

[KN] J.-P. Kahane and V. Nestoridis, Universal Taylor series and universal trigonometric series in the sense of Menchoff, J. Math. Pures Appl. 79 (2000), 855-862.

[KKN] Ch. Kariofillis, Ch. Konstadilaki and V. Nestoridis, Smooth universal Taylor series, Monatsh. Math. 147 (2006), 249-257.

[Ke] A. S. Kechris, Classical Descriptive Set Theory, Grad. Texts in Math. 38, Springer, New York, 1995.

[L] W. Luh, Approximation analytischer Funktionen durch überkonvergente Potenzreihen und deren Matrix-Transformierten, Mitt. Math. Sem. Giessen 88 (1970), $1-56$.

[MN] A. Melas and V. Nestoridis, On various types of universal Taylor series, Complex Variables Theory Appl. 44 (2001), 245-258.

[M] D. Menchoff [D. Men'shov], Sur les séries trigonométriques universelles, Dokl. Akad. Nauk SSSR (N.S.) 49 (1945), 79-82.

[N-W] C. St. J. A. Nash-Williams, On well-quasi-ordering transfinite sequences, Proc. Cambridge Philos. Soc. 61 (1965), 33-39. 
[N] V. Nestoridis, Universal Taylor series, Ann. Inst. Fourier (Grenoble) 46 (1996), 1293-1306.

[NP] V. Nestoridis and C. Papadimitropoulos, Abstract theory of universal series and an application to Dirichlet series, C. R. Acad. Sci. Paris 34 (2005), 539-543.

[P] J. Pal, Zwei kleine Bemerkungen, Tohoku Math. J. 6 (1914), 42-43.

[Se] A. I. Seleznev, On universal power series, Math. Sb. 28 (1951), 453-460 (in Russian).

[Si] J. Silver, Every analytic set is Ramsey, J. Symbolic Logic 35 (1970), 60-64.

V. Farmaki

Department of Mathematics

Athens University

Panepistemiopolis

15784 Athens, Greece

E-mail: vfarmaki@math.uoa.gr
V. Nestoridis

Department of Mathematics

Athens University

Panepistemiopolis 15784 Athens, Greece

E-mail: vnestor@math.uoa.gr and

Department of Mathematics and Statistics

University of Cyprus

1678 Nicosia, Cyprus 